\title{
Reference To Study Results Publication Name
}

National Cancer Institute

\section{Source}

National Cancer Institute. Reference To Study Results Publication Name. NCI Thesaurus.

Code C94045.

A non-unique textual identifier specifying the source of the publication identifier. 